\title{
LOGICAL SPACES FOR URBAN NOMADS
}

\author{
MR. DAVID CRAVEN, DR. NICOLA MORELLI \\ Royal Melbourne Institute of Technology \\ GPO Box 2476V Melbourne, Victoria 3001, Australia \\ david.craven@rmit.edu.aunicola.morelli@rmit.edu.au
}

\begin{abstract}
This paper examines the relevance of nomadic architecture and environments for a contemporary, global, and increasingly mobile society. The focus will be specifically on what we have named Logical Spaces. These spaces are a hybrid of real (infra)structure, products, services and information, and are transportable in as much as they can, in part, be physically re-located, as well as re-created. The elements used to define our places are no longer necessarily physical, or fixed in space.
\end{abstract}

In the first part of the paper we examine a selection of historical precedents for contemporary Logical Space, including examples of work from the Archigram studio.

In the second section we present a number of scenarios, each describing a different type of Logical Space. While many of us lead lives that are, in part, nomadic, the circumstances influencing such an existence are often varied. For some it may be a conscious decision, while for others there is simply no choice. A person may work in a nomadic fashion but return to their home periodically. Others, such as the homeless, may live completely outside the equation of house=home. An understanding of the nature of Logical Space is relevant to all of the disparate nomadic groups within our society.

Our first scenario examines the Logical WorkSpace. Here we explore what combinations of materiality, products, services and information are required to create a transportable/logical environment suitable for the contemporary worker.

The conditions required for a ubiquitous engagement with work depend, to a large degree, on technological and social change. Current technological systems are based on asymmetric communications (ie the flow of incoming information is larger than that of outgoing information from the customer) as well as nodal infrastructure. These conditions can impose limits on our ability to engage in nomadic work. From a social perspective required changes include broad revisions to the organisational models used for teamwork, cooperation and work management, which, in turn, require a new culture of work.

The other scenario examined is that of the urban homeless. Logical Spaces can provide an alternative identity and address to that traditionally associated with house and home. 


\section{Mr. David Craven \& Dr. Nicola Morelli}

What kinds of transportable/logical environments fulfil the requirements of the contemporary urban homeless? What hybrids of material, services and information constitute these environments? The projects of a number of contemporary artists working in this area will be examined, including the Refuge Wear of Lucy Orta.

In conclusion, the paper analyses and compares the positive and negative implications of the scenarios from both a utopian and dystopian perspective. Such an analysis will emphasise both the social and environmental implications related to the future development of each scenario.

\section{A Nomadic Society}

Last year Wired published an article that detailed the flying habits of a select group of New Economy types (Berger, 1999). The article was of interest not only because of its informative expose on the number of frequent flyer miles accumulated by people such as Nicholas Negroponte, but also because of its compelling demonstration that the era of the Global Citizen has well and truly arrived.

While an itinerary which sees one fly in excess of half a million miles per annum may not be normal for most of us, it serves as an, admittedly, extreme example of the fact that, ever increasingly, we live in a mobile, nomadic society.

The attraction of mobility, seemingly evident throughout society without prejudice, may be observed in our normal daily lives. As a person passes us on a scooter with improbably small wheels, we pause briefly to receive a call on our mobile, barely noticing that we are surrounded by any number of other people performing similar activities.

It is true that the above example projects a certain world-view that is not representative of all social groups in all cultures. It is however, in the author's opinion, representative of the status quo in cities as divergent as Melbourne and Kuala Lumpur.

This society is not homogenous but consists of many discreet groups, each with their own specific mobility criteria.

Nomadism may be voluntary, as in the case of someone who travels extensively because of work commitments. In this scenario, there is a strong possibility of a home base to return to. 


\section{Logical Spaces for Urban Nomads}

It may also be a lifestyle choice, perhaps as part of retirement. Selling up the family home upon retirement, and travelling around by car or boat is a dream secretly harboured by many Australians.

It is common to find this form of mobility both enabled by and dependent upon technologies of one form, or another; Mobile phone, laptop, or campervan. Almost all are mandatory accessories.

The above implies a certain socio-economic profile, one that permits a certain level of choice in decision making, control over outcomes and, most importantly, it allows one to remain in the loop, in contact, plugged in to the communications networks that are an intrinsic part of any society.

Although giving up ones home and possessions may be a voluntary act, as in the case of an Indian man who becomes a wondering holy-man, the reality of domestic nomadism is that it is often an involuntary, desperate act of someone who has lost the power to choose. The modern, urban, domestic nomad is also likely to come from a particular socio-economic profile, although one that is perhaps a long way removed from that of the nomadic worker.

While the logical workspace is largely weighted towards technology, rather than shelter, the domestic situation is quite the reverse and focuses on hardware in the form of shelter as its first priority.

As mainstream society becomes more comfortable with the Internet as a channel for information transfer, those that have traditionally been located at the periphery of the mainstream will be even further marginalised. Increasingly people who are not connected will find themselves outside of the communications loops and networks of the general society.

\section{Technological Responses to the State of Nomadism}

Throughout history, the technological tools developed by humans to support and enhance traditional nomadic lifestyles have been in the form of hardware. A wide variety of solutions to the requirement for shelter have evolved over time as specific responses to climatic and cultural differences, such as the yurt and tent.

As contemporary societies become more mobile, a dramatic increase in the number of tools available to support the new forms of nomadism has 


\section{Mr. David Craven \& Dr. Nicola Morelli}

occurred. Today there are a large number of soft, information-focused tools, although even these require some form of hardware to act as an interface.

If one were to generalise, it could be said that hard tools, being real and physical, have direct application to the provision of shelter, whereas soft tools, may be perceived as cerebral, providing access to networks, community and information.

\subsection{FROM ARCHIGRAM TO WEARABLES}

While vernacular responses to nomadic patterns of life have evolved over thousands of years there has also been, in relatively recent times, a number of speculative design propositions on this subject by a collection of significant architects, designers and artists.

No one group has been more prolific in the production of designs for the contemporary nomadic condition than the Archigram Studio. And, while their work is undoubtedly a point of reference for many papers at this conference, it is important to briefly examine a number of projects that relate to key points developed here.

The Cushicle, designed by Mike Webb in 1966-7, was to be a fully serviced, semi-autonomous nomadic unit (Cook, 1999). Fundamentally it consists of two components including a chassis, or spinal system, to provide the structural support to carry the required appliances, and an inflatable enclosure to provide shelter, along with a series of viewing screens. The enclosure and appliances could be operated independently of each other.

The identity of the Cushicle's user is left deliberately ambiguous, and could be an explorer, wanderer or other itinerant.

Mike Webb proposed the Suitaloon, conceptually an extension of the Cushicle, in 1968 (Cook, 1999). In this design the suit becomes the indivisible base unit that provides movement, a large envelope, and power. The possibility of community development is enhanced by the inclusion of a key on each suit, allowing envelopes to be grouped together, if desirable.

The same year also saw the exhibition of Milanogram, a group Archigram project, at the fourteenth Milan Triennale (Cooke, 1999). For the installation David Green produced an Inflatable Suit-Home, which could be worn, and converted into a shelter when required. The catalogue dares us to imagine hybrid interplay between hardware (visible place and objects), and software (logical, computerised systems that are unseen). 


\section{Logical Spaces for Urban Nomads}

Even though anticipating the exponential development and growth of information, or soft technologies during the past 30 years would have been difficult, it is interesting to note that the conceptual design themes first explored by Archigram back in the late sixties still appear to be fresh when compared with investigations made by contemporary architects, designers and artists.

A fashion designer turned artist, Lucy Orta has, for the better part of a decade, explored the territories of shelter, clothing, homelessness and community through projects such as 1993's Refuge Wear (Orta, 1998). This is one in a series of projects that seeks to provide design interventions for contemporary urban homelessness. The genesis of these garments, referred to as Body Architecture, lies with a desire to assist the Kurdish refugees during the Gulf War, although the solution has the potential for universal application. Made from a combination of high-tech fabrics, with details usually found on outdoor wear, the clothing is capable of significant adaptation, transforming from a full body parka, to a sleeping bag, to a tentlike shelter. Many pockets are provided to hold water, food and other essentials.

The Modular Architecture project of 1996-97 extends the themes developed for Refuge Wear, and introduces a communal dimension through the ability to combine individual units into collective shelter.

'...Four men and women may travel separately during the day, each wrapped in a waterproof, insulated, hooded body-suit made of aluminiumcoated polyamide, in which myriad pockets store water, food and medicine. At night, these people meet in a designated area - or by chance - and, after removing their body-suits, they zip them together to make a roomy, warm four-person tent. Hoods dangle at odd angles from the tent's peak, empty "legs" are pegged to the ground

The next day or during the night, after everyone has slept, they unzip the tent and climb into their body-suits to continue their travelling. Later they meet again with other people and the same tent, or another, is reconstructed.'

(Orta, 1998, pp.64-5)

Anthea Van Kopplen, a Melbourne based fashion designer, recently exhibited her LIDA project, in which a series of prototype garments for global citizens were developed (Van Kopplen, 2001). Here the focus is not specifically on the issue of homelessness as traditionally perceived, but rather on providing a range of options that extend protection and shelter 


\section{Mr. David Craven \& Dr. Nicola Morelli}

beyond the possibilities of a conventional garment. A series of zips and fasteners allow a variety of configurations that includes a cape and sleeping bag.

While the contemporary solutions so far discussed focus primarily on supplying physical shelter, there are also a growing number of tools that provide an interface to the information networks permitting the development of community within contemporary nomadic societies.

Last year a collaboration between Philips and Levis last year saw the release of the world's first commercially available electronic clothing. The jacket features an inbuilt mobile phone, as well as audio, headphones and remote control.

With its Media Lab based Wearable Computing Program, MIT is attempting to redefine the relationship between computer and user. Their aim is for computers to be worn like glasses, or clothing, and to interact with users based on the specifics of any particular context.

Technological responses to nomadism are no longer restricted to the physical, however. Completely soft products are providing solutions for the difficulties of both nomadic work and domesticity. Purely digital workspaces, such as those offered by Hot Office and Intranets, provide the opportunity for workgroups to be assembled from people in any location, at any time.

Similarly, logical communities that share a common interest, if not a geographical location, are facilitated through services such as Yahoo's EGroups.

\section{Discussion}

Interestingly, the users of the tools outlined above come from two social groups that are quite strongly differentiated by socio-economic circumstance. Of even greater interest is the relevance that these design propositions has for both groups.

Nomadic workers are currently engaged primarily in the use of soft technologies, often in order to maintain contact with a centralised head office. Shelter has not been perceived as an issue relevant to the work situation but as the requirements of business lead to an increase in travel times, the instances where short-term shelter could be of real benefit are 


\section{Logical Spaces for Urban Nomads}

increasing. Indeed, as time spent travelling grows, one could speculate that the need to maintain a traditional house/home might become questionable.

Conversely, the central issue for the homeless has been, first and foremost, one of providing physical shelter. But as population growth and economic globalisation lead to a crisis in accommodation, resulting in more people being without a home for longer periods of time, the need to provide services that extend beyond an immediate physical requirement grows.

The access to social networks provided by the soft communications technologies described above is potentially invaluable to someone who is without a home. Until recently, the lack of a physical address served as a significant impediment in remaining visible to society at large. This issue can be at least partly ameliorated by providing access to a digital address through the use of e-mail and other tools.

\subsection{IMPLICATIONS OF LOGICAL SPACE}

Propositions regarding the use of logical space, especially for the homeless, cannot be developed in a social vacuum. Any discussion must occur beyond the limits of pure technology, and also come to terms with the social and environmental issues encompassed in its application. A number of these are discussed briefly below.

\subsubsection{Social Change}

These new possibilities for work have an implied social change. The organisational models used for teamwork, cooperation and work management will require revision to accommodate the potential of new logical spaces (Morelli, 1999).

The perception of homelessness in general society needs to change. To an extent some of the possibilities discussed here will form part of the solution. A lack of fixed physical address should not result in the exclusion of individuals from the communications loops used by a society. To this end, digital addresses can assist in keeping people in the network, and in making them part of a community.

\subsubsection{Technological Robustness and Asymmetry}

An issue of particular relevance to software tools is the robustness of a technology and its ability to imposes limits on its potential to provide ubiquitous access (Morelli, 1999).

Likewise, A serious issue for nomadic workers is asymmetrical data-flow, where upload speeds are significantly slower than those for download. This 


\section{Mr. David Craven \& Dr. Nicola Morelli}

condition has the potential to result in the development of information rich nodes, with corresponding data deserts, rendered geographically, in realtime.

\subsubsection{Access to Hardware/Interface}

It would seem reasonable to suppose that there is some correlation between being information and cash rich and, conversely, between the information poor and the just plain poor!

How is access provided to the various components of logical space? How for example, in the case of hardware, could the homeless possibly afford the high performance shelters proposed by Lucy Orta, or Anthea Van Kopplen?

Also, the provision of access to soft technologies alone will not ensure their equitable use. Invariably access is obtained through an interface that is a hard/software hybrid.

In the case of the homeless in particular, important questions arise when considering the nature of an interface. How will access to the interface be provided and funded, and how can it be ensured that users understand the interface's functionality?

The case of access for nomadic workers is currently, at least, more clearly established. A number of methods including neighbourhood work centres, and completely mobile work units, are already relatively established (Morelli, 1999). Less clear are the possibilities of future work scenario where employees spend virtually no time at head office, but are constantly involved in work-related travel.

\subsubsection{Utopia/Dystopia}

While in an ideal world the condition of homelessness would not exist, we need to accept that, at least in the short term, a significant proportion of the world's population will be homeless at any particular point in time. In the present situation, logical spaces may be a way to provide a valuable service to people.

In which case, one could ask the question, how useful is a digital address when you are homeless? Perhaps, indeed, it is of limited relevance, as it plays no part in providing for the immediate requirement of physical shelter. It does, however, assist in supplying access to information/networks, with the inherent potential of participating in a larger community.

As global business expansion is facilitated by the growth of the Internet and World Wide Web, the need for high-level face-to-face meetings is actually 


\section{Logical Spaces for Urban Nomads}

increasing. This means that some employees are travelling more than ever (Berger, 1999).

While the imagery of the digital briefcase and global portable shelter convey a message of the freedom to work wherever and whenever (with the subtle implication of an increase in leisure time), the unfortunate reality would seem to be that, in the modern workplace, when people are given freedom to choose their working conditions, there is a tendency to towards working more rather than less, causing the barrier traditionally drawn between work and leisure to become blurred.

Under these circumstances, traditional relationships can be put under significant stress, and may become compartmentalised by geographic location (Berger, 1999).

\subsubsection{Sustainability}

As well as a consideration of the social issues, it is necessary to understand the environmental implications of a nomadic society. Will this new world of work and domesticity be more sustainable than our previous and current patterns?

On the one hand, workers may choose to travel only when necessary, rather than as a twice-daily ritual. Work may become an activity that is conducted largely from, or nearby to home.

However, an increase in the mobility of high-level executives, with an associated rise in the numbers of plane-flights and car journeys will inevitably have a negative environmental impact.

On the other hand, a logical space providing both shelter and the ability to communicate may potentially reduce the need to constantly return to the home base at the completion of every assignment.

The sustainability of logical space for the homeless provides a different environmental scenario. Due to the costs associated with travel over long distances, it is more likely that the homeless will remain located primarily within the same urban environment, and it is therefore unlikely that their contribution to ecosystem degradation would compare significantly with that of the high-flying business executive. For the homeless the sustainability implications are more likely to be social, rather than environmental. 


\section{Mr. David Craven \& Dr. Nicola Morelli}

\section{Conclusion}

Whether it is the growth in the number of homeless in the streets of our cities, or an increasing number of executives living in airports, it would seem that one of the symptoms of a globalised society is that more people will be leading lifestyles that are, at least to a significant degree, nomadic.

Transportability can consist of either logical recreation of an environment, or its physical relocation. It would seem that determining whether your transportable environment is recreated or relocated depends to a large degree on who you are and what your socio-economic position is.

The nomadic worker tends to rely on the hard and software necessary to provide access to virtual work environments. The issue of shelter in this context is secondary. While to the urban homeless, the issue of real, tangible shelter is paramount.

Perhaps physical and logical spaces are in fact complimentary, with each enhancing the experience of the other. And maybe the mental image of a dot.com executive sleeping in body architecture in the airport waiting-lounge is not as far removed from a homeless person receiving e-mail on their mobile phone, after all.

\section{References}

Berger, W.: 1999, Life Sucks and Then You Fly, Wired, 7(08) 156-163.

Cook, P. (ed.): 1999, Archigram, revised edition, Princeton Architectural Press, New York.

Morelli, N.: 1999, Future Configurations for Remote Work, Foresight. The Journal of Future Studies, Strategic Thinking and Policy, 1(03), 251-264.

Orta, L.: 1998, Process of Transformation, Editions Jean Michel Place, Paris.

Van Kaplan, A.: 2001, LIDA, RMIT Masters by Project examination exhibition. 